\title{
Il castello di Morrea. Evoluzione e destino delle strutture fortificate nel centro Italia
}

The castle of Morrea. Evolution and destiny of fortified structures in Central Italy

\author{
Stefano Cecamore \\ Dipartimento di Architettura - Università degli Studi dell'Adriatico “G. d'Annunzio" Chieti-Pescara, Pescara, Italy, \\ stefano.cecamore@unich.it / stefanocecamore@gmail.com
}

\begin{abstract}
The shape that today characterizes the fortified building of the castle of Morrea is only the last of the various stratifications that have modified the original layout thorughout the centuries. The current aspect of the building is most likely linked to the interventions promoted by the Piccolomini family between the twentieth and twenty-first century. The building represents the evolution from castrum to aristocratic residence that involves the various fortified structures placed along the Apennine ridge between the eastern and western front of Central Italy.

In this area the various degrees of transformations of the castles, which are periodically updated for reasons due to oxidation and representative natures, are clearly readable.

The artifacts analyseable represent a wide range of samples of fortifications of the most ancient form of specialized buildings which were often largely left in the state of ruins, including that of buildings yet still functional, however, far from their consistency and original purpose.

The overall panorama of this architectural heritage outlines a complex scenario consisting of problems related to the conservation and maintenance where restoration projects need to find and be in the proper position of restoration and respect of the bond between the building environment, the landscape and the identities of the territory.
\end{abstract}

Keywords: Fortified historical centres, abandon, traditional building techniques, landscape.

\section{Introduzione}

I crinali appenninici del centro Italia rappresentano da sempre un territorio di difficile penetrazione le cui caratteristiche morfologiche condizionano sensibilmente la possibilità di stabilirvi insediamenti umani stanziali tanto da ritardare lo sviluppo di veri e propri impianti fortificati, chiaramente circoscritti, fino al IV secolo a.C. La rete degli insediamenti riconducibili alle genti originarie del centro Italia, identificati nelle fonti di época romana come oppida e castella, risultano posti in posizione strategica e in colle-

gamento visivo tra loro, secondo una trama che definisce una primordiale struttura di controllo del territorio che trova forma compiuta ed evolve in un sistema chiaramente riconoscibile intorno al X-XI secolo. La dominazione normanna risolve la complessa organizzazione politicoterritoriale attraverso un nuovo assetto dei centri di crinale del centro Italia sfruttati quali punti di confine tra i regni meridionali e lo Stato della Chiesa. I numerosi castra e impianti fortificati alternativamente distrutti e potenziati lungo la 
frontiera con il Patrimonium Sancti Petri delineano un vasto patrimonio di testimonianze materiali dell'evoluzione storico-architettonica di questo territorio.

\section{Le origini di una "Terra di confine"}

Il ruolo di frontiera tra Stato della Chiesa e regni del sud è definitivamente sancito dall'intento di Federico II di imprimere un'impronta egemonica su feudalità, clero e società del Mezzogiorno d'Italia, perseguito con le assise di Capua del 1220 (Fonseca, 1998, p. 14; Clementi, 1999, p. 231).

Con le sanctiones previste in base alla costituzione de resignandis privilegiis (Tramontana, 1986 , p. 232) l'imperatore svevo avvia il processo di revisione di tutti i titoli di possesso di beni e privilegi della feudalità locale imponendo anche lo smantellamento di castelli e fortezze costruiti abusivamente e la riannessione al demanio regio delle restanti, autorizzate in passato dalla legislazione normanna.

La nuova rete sveva di controllo del territorio, strutturata in passi e fortilizi d'altura posti a sorvegliare le principali vie di transito, definisce un sistema di governo e gestione centralizzata che quest'area dell' Appennino centrale aveva conosciuto solo molti secoli prima con la dominazione romana.

Infatti la sottomissione della gens aequa e dei marsi e la loro successiva latinizzazione tra IV e I secolo a.C. trova definitiva risoluzione con la fondazione dei tre principali municipia del territorio, Carsioli, Alba Fucens e Marruvium e il tracciamento e potenziamento della Claudia Valeria organizzata nei due tratti principali da Roma a Tibur e da Tibur a Ostia Aterni e nelle sue diramazioni nell' Ager Albensis e verso gli attuali Piani Palentini e Val Roveto.

Poco resta degli impianti pre-romani, non sempre recuperati e riutilizzati per la fondazione di nuovi avamposti di controllo e gestione del territorio; le strutture superstiti testimoniano il ricorso frequente ad opere poligonali in calcare locale comunemente utilizzate nelle recinzioni dei siti fortificati e rilevabili in sede di scavi archeologici (Somma, 2000, pp. 26-27).
In età arcaica (VI secolo a.C.) si assiste alla definitiva diffusione degli ocres d'altura in tutta l'area marsicana sviluppatisi a partire dalla seconda età del Ferro (VIII-VII secolo a.C.); piccole città-stato riferibili ad una società guerriera ad economia diversificata (agricoltura, pesca, caccia, metallurgia, allevamento) sono identificabili nei centri fortificati su fondazioni rocciose, cinti da apparati murari difensivi in opera poligonale montata a secco utilizzando grandi e medi blocchi di calcare.

Una vera e propria militarizzazione del territorio è, però, ascrivibile all' arco temporale che, tra V e IV secolo a.C., assiste allo sviluppo dei grandi centri distrettuali di matrice marsa ed equa: $A n$ tinum nella Val Roveto, Milionia nella Valle del Giovenco, Alba nei Piani Palentini, Carseoli nella Piana del Cavaliere (Grossi, 2011, p. 188).

Recenti studi (Leo, et al., 2011, pp. 53-60) intrapresi al confine col territorio laziale ampliano il bagaglio di conoscenze sui centri di aggregazione del periodo italico individuabili sul fronte occidentale marsicano; il sito, individuato presso la località di Santo Stefano frazione di Sante Marie (AQ) a 1149 m.s.l.m. presso il declivio vallivo identificato dai Monti Carseolani e dai Monti Faito e San Nicola, presenta tracce ancora rilevabili di una cerchia muraria le cui caratteristiche dimensionali sono ipotizzabili in base allo studio dei lacerti murari -posti in corrispondenza dell' accesso nord- conservati fino ad un'altezza massima di $2 \mathrm{~m}$. La struttura presenta un peculiare apparecchio in pietra calcarea locale, organizzato in elementi dimensionalmente disomogenei (lunghezza tra 0,40-1,5 m) appena sbozzati e montati a secco a formare un paramento apparentemente privo di piani di posa e coadiuvato strutturalmente dall'inserimento di zeppe negli interstizi della cortina muraria. In linea generale questo recinto si configura come un abitato protetto di piccole-medie dimensioni non dissimile dalle tipologie delle fortificazioni d'altura riscontrabili in ambito italico.

Gli impianti pre-romani con connotazioni difensive e stanziali disseminati in territorio equo, da sempre sensibile frontiera tra l'Appennino orientale e quello occidentale, tracciano una mappa dei siti -individuati dagli studi condotti nei terri- 
tori limitrofi ai fiumi Turano, Salto, Imele ed Aniene (D'Ercole, Martellone, 2004, p. 32) (Fig. 1) - disegnata da circa cinquanta insediamenti d'altura, alcuni dei quali presentano strutture fortificate plurime -ascrivibili al V secolo a.C.come quelle leggibili in località Monte San Felice e Monte San Nicola poste in corrispondenza dell'attuale città di Scurcola con chiaro intento di controllo e presidio del territorio all'imbocco della Valle dell'Imele.

L'acuirsi dei conflitti tra romani e gens Aequa tra V e IV secolo a.C. determina, invece, l'infittirsi di oppida in corrispondenza dell'alta Valle dell'Aniene, estremo lembo occidentale al confine col Latium vetus (Bianchini, 2011, pp. 21-27).

La definitiva sottomissione delle popolazioni autoctone, individuate dai romani come tribù Aniensis, è sancita dall'istituzione delle due colonie latine di Alba Fucens -303 a.C.- e Carsio$l i$, tra il 302 e il 298 a.C. Secondo quanto riportato da Tito Livio (Ab Urbe Condita, IX, 45) gli scontri tra romani ed equi, successivi alla seconda guerra sannitica, comporta la presa e distruzione di 31 oppida equi; i primitivi nuclei fortificati dalle popolazioni autoctone risultano completamente distrutti e abbandonati e solo in minima parte coinvolti nel processo di adattamento al modello insediativo urbano romano.

In tutti i territori equi (D’Ottavi, 2010, pp. 4143) e marsicani il processo di romanizzazione comporta la crisi del sistema insediativo fortificato degli oppida e la diffusione dei vici e successivamente dei municipia che raramente impongono una riconversione urbana agli originari avamposti d'altura, come nel caso di Antinum (odierna Civita d'Antino) nella Val Roveto o di Lucus Angitie a nord dell'attuale Luco dei Marsi (Somma, 2000, p. 29).

Il ritorno agli insediamenti d'altura è legato al successivo fenomeno dell' "Incastellamento" ed è testimoniato dai molti siti fortificati di quest'area dell' Appennino centrale accomunati da impianti databili tra XI e XII secolo, il cui elemento ricorrente appare la torre primigenia con funzione di avvistamento.
Le successive evoluzioni, che ampliano e modificano gli impianti originari, aprono il campo a nuove tipologie castrali; a quella comunemente indicata in letteratura come "castello-recinto" si affiancano realtà complesse che associano il castello-residenza al borgo murato o il recinto castrale a cerchie di "case-mura" fino all'integrale sostituzione delle preesistenze con nuove fabbriche non dissimili dalla rocca comunemente intesa.

Il panorama costruttivo delineato dallo studio delle strutture in elevato raccoglie le tracce delle principali trasformazioni dei sistemi fortificati: dai recinti castrali allo stato di rudere, passando per quelli sopravvissuti all'abbandono e inglobati nei sistemi urbani, fino agli impianti rivisitati in chiave rinascimentale e ai castelli residenza delle grandi casate romane.

\section{Dalla torre al palazzo}

Alle famiglie Orsini, Colonna e Barberini sono dovuti, infatti, i più incisivi interventi di trasformazione e rifondazione dei castelli di questa "terra di frontiera" -oggi segnata dal confine tra Abruzzo e Lazio- che tra XV e XVII secolo modificano sostanzialmente alcuni impianti fortificati di origine medievale.

In área marsicana, in particolare, i tratti distintivi di queste opere di rilettura e adattamento dei $c a$ stra originari alle sopraggiunte esigenze di natura ossidionale e rappresentativa del XV secolo, si colgono nel primitivi bastioni della rocca di Tagliacozzo (La Valle, 2013, p. 392) e nell'impianto antropomorfo tardoquattrocentesco di quella di Scurcola (Salciccia, 2000 , pp. 44-50); in questo caso il nuovo apparato difensivo inviluppa le preesistenze in una struttura che lega forma e funzionalità del manufatto in osservanza ai dettami costruttivi di Francesco di Giorgio Martini del quale non si può escludere una frequentazione dei borghi marsicani intorno al 1490 (Ghisetti Giavarina, 1987, pp. 99-101).

La trasformazione in palazzo del castello di Morrea (Figg. 1-2), promossa dai Piccolomini, dopo il 1489 -data di acquisizione per via matrimoniale del feudo- rappresenta, invece, la 
forma di stratificazione più evoluta delle "residenze-fortezza".

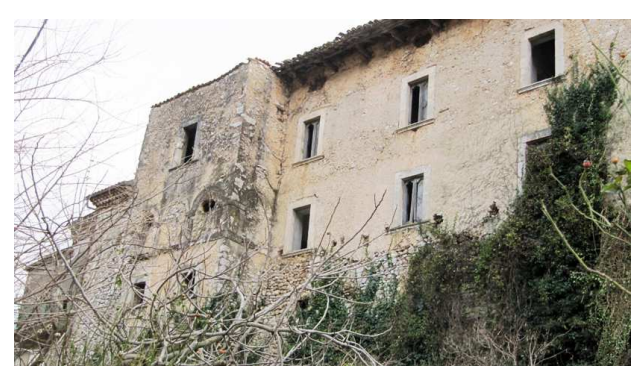

Fig. 1. Morrea, fronte palaziato (Autore, 2012).

Del castrum primitivo (Fig. 3) -uno dei cinque che le fonti relative all' area marsicana collocano in corrispondenza di fortificazioni pre-romaneposto nel lembo nord-occidentale del centro storico, si conservano i profili scarpati del fronte est e le torri circolari oltre a poche testimonianze delle successive fasi pre-rinascimentali. La datazione orientativa delle fasce di muratura superstite delle torri e la mancanza in tutti i corpi di fabbrica scarpati del redondone confermano l'appartenenza dei volumi basamentali ad una fase temporale anteriore al XV secolo (Somma, 2000, p. 72).

L'impronta distintiva dell'avvenuta metamorfosi da castello a residenza palaziata è evidente nel prospetto principale della corte interna organizzato in una sovrapposizione di volumi pieni e vuoti aperti in archi a tutto sesto a livello della loggia del piano nobile (Fig. 4).

Ulteriori tracce del complessivo riassetto del fortilizio in chiave tardo rinascimentale si colgono nel lessico architettonico di singoli elementi costruttivi, nell'omologazione delle mostre di portali e finestre e nella revisione degli orizzontamenti dei vani principali, trasformati da semplici impalcati in solai con doppia orditura e tavolato ligneo rivestito con carta decorata ad imitazione di trame e tessuti da parati.

Il progressivo abbandono del complesso, innescato al principio del XIX secolo dall'abolizione del feudalesimo -che relega Morrea al ruolo marginale di frazione di Civita D'Antino, prima, e di San Vincenzo Valle Roveto, poi- e amplificato dai danni inferti all'abitato dal terremoto della Marsica del 1915, arresta le dinamiche di trasformazione del castello promosse nei secoli precedenti.

La perdita di una residenzialità stanziale del centro storico comporta quella relativa della consuetudine all'uso e alla manutenzione dei manufatti che, se da un lato mostra tra le conseguenze più gravi quella dell'evidente stato di degrado delle preesistenze, ha dall'altro il merito inconsapevole di aver scongiurato alterazioni formali e sofisticazioni in stile dell'impianto castrale originario.

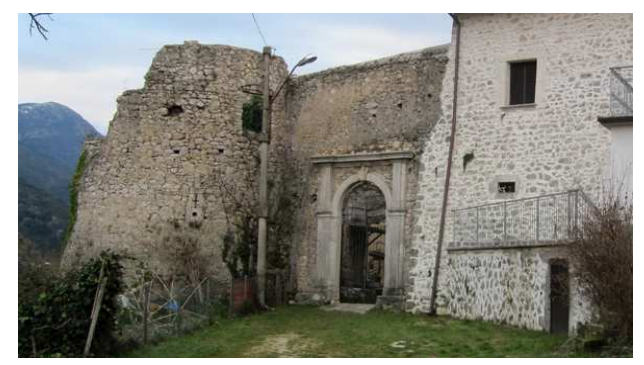

Fig. 2. Morrea, ingresso alla corte principale; resti di una torre circolare (Autore, 2012).

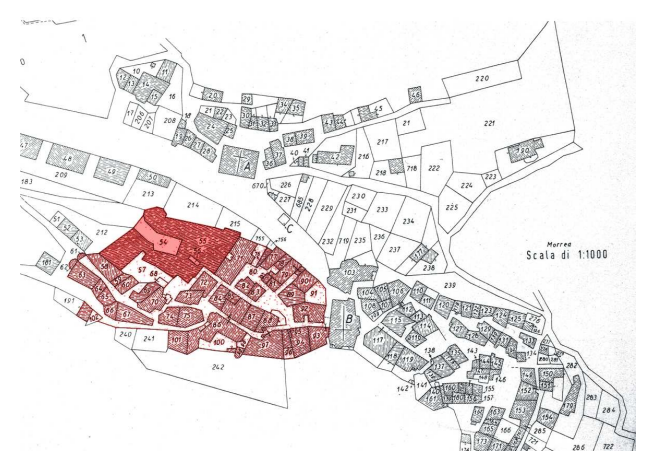

Fig. 3. Morrea, tessuto edilizio del castrum originario.

L'inevitabile perdita delle funzioni specialistiche e dell'identità militare di queste strutture comporta, infatti, un ventaglio di molteplici sorti per castelli e recinti castrali.

All'alba del XX secolo gli esiti della metamorfosi politico-territoriale del centro Italia vanno dal completo abbandono dei manufatti, seguito dalla conseguente ruderizzazione, al riutilizzo e adattamento delle preesistenze declinati secondo nuove vocazioni pubblico-private che si spingo- 
no dal semplice frazionamento dei corpi di fabbrica -come nel caso di Morrea- alla loro complessiva riconfigurazione.

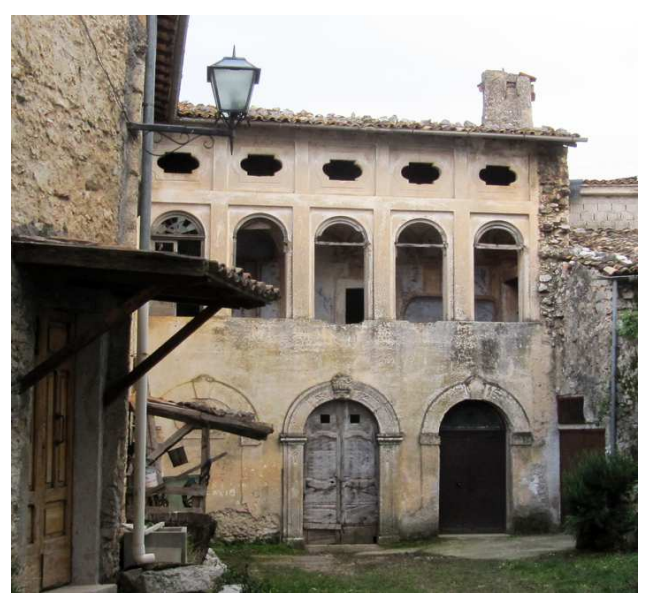

Fig. 4. Morrea, corte interna; loggia del piano nobile (Autore, 2012).

E' il caso del castello di Collalto Sabino (RI) che raccoglie nelle sue vicende costruttive di lunga durata, oltre alle testimonianze materiali dell' "incastellamento", una fase palaziale -(XVIXVII secolo) legata alle nobili famiglie Soderini e Barberini- rivisitata tra XIX e XX secolo in chiave eclettica attraverso un frainteso medievismo funzionale all'attuale destinazione turisticoricettiva (Figg. 5 e 6).

Resta fortunatamente inalterato il rapporto con il tessuto urbano del centro storico e con il paesaggio circostante come nel caso del castello di Pereto nel quale gli interventi effettuati durante il secolo scorso sui principali corpi di fabbrica, dovuti ad un adeguamento residenziale del manufatto, non ne hanno compromesso sensibilmente forma e struttura, lasciando anche spazio ad importanti campagne di analisi e catalogazione dei paramenti murari del mastio e delle mura del centro storico e alle contestuali opere di restauro (Branciani, 2008).

L'impianto, planimetricamente complesso, conserva testimonianze riferibili a molteplici fasi costruttive e raccoglie per successive evoluzioni i vari passaggi che determinano, tra XII e XIV secolo, la trasformazione della primigenia fase castrale (torre e recinto) in residenza stanziale con un elementare nucleo palaziato.

La fondazione quale avamposto di guardia apicale destinato al controllo della sottostante Piana del Cavaliere lo accomuna ai vicini centri fortificati di Celle/Carsoli e di Oricola.

I tre "castelli", oltre all'evidente nesso territoriale che li vede quali maggiori punti focali del territorio carseolano, sono strettamente legati da componenti storico-identitarie e dal ruolo di fondamentali testimonianze della cultura costruttiva locale chiaramente derivata dalla "civiltà della pietra" e dallo sfruttamento delle risorse locali. Il ricorso alla pietra calcarea quale principale materiale da costruzione rappresenta, infatti, una costante dell'edilizia militare, civile e religiosa del territorio; campagne di scavo nei siti più antichi, operazioni di rilievo su fabbriche medievali e rinascimentali e le costruzioni del tessuto urbano più recente dei centri minori, ne confermano un uso costante declinato in elementi lavorati, semilavorati o erratici e di recupero.

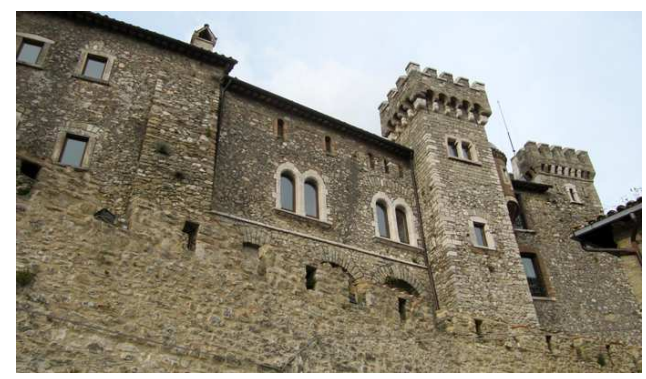

Fig. 5. Collalto Sabino, castello (Autore, 2014).

Nel caso di Oricola il ruolo di controllo del territorio è evocato già nell' etimologia del toponimo tramandata dalla tradizione locale (auricola/orecchio) e nella configurazione dello stemma comunale: due padiglioni auricolari sormontati da una stella azzurra e sospesi su tre colli verdeggianti (Lapenna, 2003, p. 17; Amici, 2009, p. 11). Il centro storico, posto a 809 m.s.l.m., partecipa quale "porta d'Abruzzo" al complesso sistema di avvistamento e presidio degli attraversamenti vallivi appenninici ereditato dagli avamposti italici e pre-romani posti in collegamento visivo tra loro. 


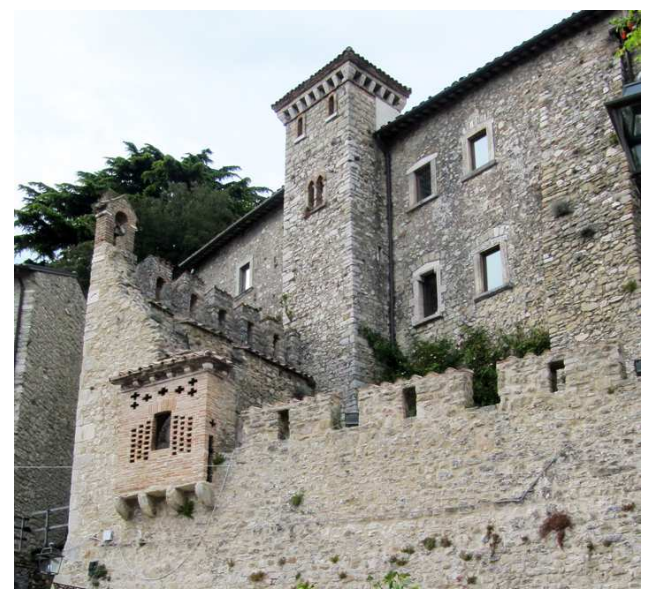

Fig. 6. Collalto Sabino, castello (Autore, 2014).

Il castello organizzato secondo un impianto triangolare con torri cilindriche ai vertici è riconducibile a un'integrale ricostruzione di epoca basso medievale (XV secolo); non è nota la possibile collocazione temporale di questo intervento, ma in ogni caso il paramento murario delle torri, variamente disturbato da numerosi interventi di consolidamento, è assimilabile ad una tipologia ricorrente in corpi di fabbrica circolari conformati in apparecchiature a filari-composte da pezzame calcareo essenzialmente omogeneo e prive di frammenti fittili- riferibili ad ambiti cronologici non anteriori al XIII secolo (Somma, 2000 , p. 92). Le devastazioni riportate in letteratura, riferibili all'assedio e alla distruzione della vicina Carsioli alla metà del XIII secolo (Amici, 2009 , p. 35) e della stessa Oricola al principio del XVI (Mancini, 2003, p. 84), devono presumibilmente aver contribuito all'alterazione sostanziale del centro storico e del castello sottoposto, anche, in tempi recenti alla totale revisione degli interni convertiti ad uso civico e residenziale.

Lo stato di avanzata ruderizzazione e di parziale abbandono del borgo antico -ricordato nelle fonti come castrum di Celle- probabilmente conseguente a eventi calamitosi lontani nel tempo (Santoro, 2001, p. 34), proietta, invece, l'insediamento urbano di colle Sant'Angelo oggi Carsoli- fuori dall'orbita degli adeguamenti funzionali e strutturali del XX secolo. Le principali alterazioni riguardano l'impianto apicale del castello, la porta de' Merli, demolita con il relativo tratto muraio di collegamento con porta $\mathrm{Na}$ poli nell'abitato a valle, i vuoti urbani provocati dai bombardamenti e i crolli localizzati di solai e coperture dovuti alla mancata manutenzione.

Si distinguono due diversi perimetri fortificati tracciati in accordo con le naturali caratteristiche orografiche del sito- e predisposti in modo da configurare un anello difensivo intorno al primordiale avamposto di guardia. Lo studio dei paramenti e dei lacerti murari indirizza verso la prefigurazione di due campagne di manutenzione e ammodernamento del costruito precedenti agli eventi sismici del XV secolo, probabilmente coincidenti con quelle citate in letteratura (Sthamer, 1995, p. 118). A una prima sostanziale revisione degli apparati difensivi operata in época sveva, identificabile nel recinto sommitale inquadrato da torri quadrangolari cave, sarebbe seguito il potenziamento e l'allargamento del perímetro fortificato promosso da Carlo II d'Angiò.

L'analisi dei caratteri costruttivi ricorrenti nell'edificato, organizzato radialmente a partire dall'emergenza sommitale del recinto castrale, evidenzia una sostanziale conservazione del tessuto di matrice medievale; non mancano però episodi architettonici riferibili a periodi successivi che testimoniano la contunuità nell'uso abitativo almeno di una buona percentuale del costruito.

Mostre di porte e finestre delle case-mura presentano stilemi di indirizzo rinascimentale e la riconfigurazione interna dell' aula della Chiesa di Sant'Angelo, oggi allo stato di rudere, che un'iscrizione attribuisce alla famiglia De Leoni è riconducibile a un intervento successivo al XVIXVII secolo.

A differenza dell'insediamento fortificato di Carsoli, numerosi manufatti in area marsicana presentano perimetri variamente fortificati -con torri interne o giustapposte al circuito murario- e strutturalmente stratificati, che per contingenze legate ad eventi calamitosi o mancate revisioni ossidionali e formali, non mostrano, però, tracce di fasi costruttive posteriori al XIII secolo e i relativi passaggi da architettura prettamente specialistica (militare) a usi diversificati. 
Tra quelli annoverati nel Catalogus Baronum, Entremontes, oggi Tremonti rappresenta uno degli esempi più significativi di torre apicale (1120 m.s.l.m.) coadiuvata da un recinto fortificato il cui perimetro irregolare segue la morfologia del sito e presenta ai vertici ulteriori torri geometricamente diversificate.

Il paramento della torre centrale, impostata su pianta quadrata con lato di circa $5,5 \mathrm{~m}$ e conservata per un'altezza massima di $6 \mathrm{~m}$, rivela due distinte fasi costruttive: una caratterizzata da blocchetti calcarei regolarizzati posti in opera in filari orizzontali -databile al XII-XIII secolo- e una fase anteriore identificata da pezzame di calcare di dimensioni molto irregolari e rari frammenti fittili che ne anticipa la datazione al X-XI secolo in epoca pre-normanna (Somma, 2000, pp. 84-85).

La torre circolare dal lieve profilo "a scarpa", distaccata dal recinto principale sul versante settentrionale -di probabile matrice angioina- trova invece assonanze con le torri del recinto superiore del castello di San Donato.

Quest'ultimo, ubicato sullo sperone sudorientale del monte Faito, si configura come un sistema fortificato complesso, posto in collegamento visivo con i castra di Castelvecchio, Tremonti, Civita di Tagliacozzo e Girifalco; i numerosi manufatti fortificati che lo definiscono delineano successive fasi costruttive che spaziano dall'XI al XII secolo e la conformazione di quello apicale lascia presupporre una possibile destinazione dello stesso a residenza signorile.

L'attuale condizione di rudere non permette, però, di rintracciare elementi utili a suffragare l'ipotesi dell'esistenza nel complesso di San Donato di volumi residenziali rintracciabili, invece, a Pereto nei 5 vani sovrapposti interni all'imponente mastio svettante nell' area del $\mathrm{ca}$ strum sommitale la cui principali fasi costruttive sono riferibili ad un arco temporale a cavallo tra dominazione normanna e sveva (Branciani, 2008, p. 117).

\section{Conclusioni}

Il contributo raccoglie alcune considerazioni frutto di campagne d'indagine speditive sul patrimonio fortificato della dorsale appenninica tra Abruzzo e Lazio.

Le brevi note sui casi citati sono preliminari a future e più estese ricognizioni nei territori di frontiera che presentano una casistica ampia di episodi variamente stratificati.

Il castello di Morrea si pone, infatti, in una posizione intermedia nella scala di conservazione/trasformazione dell'architettura castrale distribuita tra tra la ruderizzazione e il completo abbandono e lo smantellamento e l'alterazione irreversibile delle preesistenze.

Accostarsi allo studio di questi manufatti allo stato di rudere, ubicati in aree marginali e suburbane, o di edifici presenti nei centri storici stravolti nella loro consistenza materiale, presuppone che il percorso metodologico di analisi e di intervento sia mediato dalla consapevolezza della loro natura metamorfica e dalla necessità di comprenderne lo stato di transizione tra le condizioni di relitto, rudere e rovina (Doglioni, 2011), a volte leggibili in forma compiuta, più spesso difficilmente distinguibili nell'ambito dello stesso palinsesto architettonico.

Nel caso delle fortificazioni presenti nell'Appennino centrale e in Abruzzo in particolare, qualunque intervento di recupero e valorizzazione non può prescindere, oggi, dallo strettissimo legame tra manufatti e territorio -che a volte raggiunge la totale simbiosi del "paesaggio totale" (Serafini, 2016, p. 130)- e dalla loro condizione di "architetture fragili" (Varagnoli, 2016, p. 135) nate come strutture specialistiche e oggi orfane delle funzioni militari e difensive che le hanno generate.

\section{Bibliography}

Bianchini, M. (2001). Edilizia storica della Marsica occidentale, Dedalo, Roma.

Clementi, A. (1999). "L’Abruzzo in epoca sveva", in Fonseca, C.D. ed., Atti del convegno internazionale di Studio promosso dall'Istituto Internazionale di Studi federiciani. Consiglio Nazionale delle Ricerche: Mezzogiorno - 
Federico II- Mezzogiorno, (Potenza - Avigliano - Castel Lagopesole - Melfi, 18-23 ottobre 1994), Roma, pp. 231-251.

D’Ercole, V.; Martellone, A. (2004). "Gli Equi prima della conquista romana”, in Lapenna, S. ed., Gli Equi tra Abruzzo e Lazio, Sulmona, pp. 31-59.

D’Ottavi, P. (2010). "Il territorio degli Equi o Equicoli”, Il foglio di Lumen, 27, pp. 41-43.

Doglioni, F. (2011). Nel Restauro. Progetti per le architetture del passato, Marsilio, Vicenza.

Fonseca, C.D. (1998). “«Castra ipsa possunt et debant reparari»: attività normativa e prassi politica di Federico”, in Atti del convegno Internazionale di Studi Federiciani. Consiglio Nazionale delle Ricerche: "Castra ipsa possunt et debant reparari”. Indagini conoscitive e Metodologie di Restauro delle Strutture Castellane Normanno-Sveve, (Castello di Lagopesole, 16-19 ottobre 1997), Roma, pp. 13-22.

Ghisetti Giavarina, A. (1987). "Fonti documentarie e letture di fabbriche: Francesco di Giorgio Martini in Abruzzo", in Spagnesi, G. ed., Atti del XXI Congresso di Storia dell'Architettura (Roma 1983), Roma.

Grossi, G. (2011). "Sintesi sulla storia dell'area marsicana dall'età del Ferro al Medioevo”, in Gossi, G.; Irti, U. eds., Carta Archeologica della Marsica, Avezzano, vol.1, pp. 181-234.

Leo, A. De; Stefano, S. Di; Nervo, A. (2011). "Ricerche presso il sito fortificato d'altura di Santo Stefano nel territorio comunale di Sante Marie (AQ), località Le Scalette", Quaderni di Archeologia d'Abruzzo, 1, pp. 53-60.

Salciccia, M. (2000). La rocca Orsini di Scurcola marsicana. Note e studi su un monumento del Rinascimento italiano, Pescara.

Santoro, L. (2001). "I castelli d'Abruzzo nell'evoluzione dell'architettura difensiva", in Abruzzo dei castelli. Gli insediamenti fortificati abruzzesi dagli italici all'Unità d'Italia, Ascoli Piceno, p. 134.

Serafini, L. (2016). "Paesaggi di castelli e viceversa. Il caso abruzzese”, in Castagnolo, V.; Cadilhac, R. de; Perfido, P.; Rossi, G. eds., Atti delle giornate di studio: Architettura Fortificata. Rilievo e Restauro, (Politecnico di Bari, 7-9 aprile 2016), Martina Franca, pp. 127-134.

Somma, M.C. (2000). Siti fortificati e territorio. Castra, castella e turres nella regione marsicana tra X e XII secolo, Palombi, Roma.

Sthamer, E. (1995). L'Amministrazione dei castelli nel Regno di Sicilia sotto Federico II e Carlo I d'Angiò, Martina Franca, p. 118.

Tramontana, S. (1986). La monarchia normanna e sveva, Torino.

Valle, M. La. (2013). "Un insediamento fortificato di altura nella Marsica tra XII e XVII secolo: le strutture sul monte Civita di Tagliacozzo (AQ)", Archeologia Medievale, XL, pp. 387-413.

Varagnoli, C. (2016). "L'avvenire dei castelli: riflessioni ed esperienze”, in Castagnolo, V.; Cadilhac, R. de; Perfido, P.; Rossi, G. eds., Atti delle giornate di studio: Architettura Fortificata. Rilievo e Restauro, (Politecnico di Bari, 7-9 aprile 2016), Martina Franca, pp. 135-142. 